\title{
Initial Time Difference Stability of Causal Differential Systems in terms of Lyapunov Functions and Lyapunov Functionals
}

\author{
Coşkun Yakar ${ }^{1}$ and Mustafa Bayram Gücen² \\ ${ }^{1}$ Department of Mathematics, Gebze Technical University, Gebze, 141-41400 Kocaeli, Turkey \\ ${ }^{2}$ Department of Mathematics, Faculty of Sciences, Yildiz Technical University, Istanbul, Turkey \\ Correspondence should be addressed to Coşkun Yakar; cyakar@gyte.edu.tr
}

Received 9 June 2014; Revised 10 November 2014; Accepted 24 November 2014; Published 22 December 2014

Academic Editor: Zhilong L. Huang

Copyright @ 2014 C. Yakar and M. B. Gücen. This is an open access article distributed under the Creative Commons Attribution License, which permits unrestricted use, distribution, and reproduction in any medium, provided the original work is properly cited.

\begin{abstract}
We investigate the qualitative behavior of a perturbed causal differential equation that differs in initial position and initial time with respect to the unperturbed causal differential equations. We compare the classical notion of stability of the causal differential systems to the notion of initial time difference stability of causal differential systems and present a comparison result in terms of Lyapunov functions. We have utilized Lyapunov functions and Lyapunov functional in the study of stability theory of causal differential systems when establishing initial time difference stability of the perturbed causal differential system with respect to the unperturbed causal differential system.
\end{abstract}

\section{Introduction}

Lyapunov's second method is a standard technique used in the study of the qualitative behavior of causal differential systems along with a comparison result [1] that allows the prediction of behavior of a causal differential system when the behavior of the null solution of a comparison system is known. However, there has been difficulty with this approach when trying to apply it to unperturbed causal differential systems and associated perturbed causal differential systems with an initial time difference. The difficulty arises because there is a significant difference between initial time difference (ITD) stability and the classical notion of stability. The classical notions of stability are with respect to the null solution, but ITD stability is with respect to unperturbed causal differential system where the perturbed causal differential system and the unperturbed causal differential system differ in both initial position and initial time [2-4].

In this paper, we have resolved this difficulty and have a new comparison result which again gives the null solution a central role in the comparison differential system. This result creates many paths for continuing research by direct application and generalization.
In Section 2, we present definitions and necessary background material. In Section 3, we discuss and compare the differences between the classical notion of stability and the recent notion of initial time difference (ITD) stability. In Section 4, we have comparison theorems via Lyapunov functions with initial time difference. In Section 5, we have our main results of initial time difference stability criteria and asymptotic stability via Lyapunov functions and we finally have our main result of initial time difference uniformly asymptotic stability via Lyapunov functionals.

\section{Preliminaries}

In order to investigate the theory of stability for causal differential equations, we need comparison results in terms of Lyapunov-like functions.

Consider the causal differential systems

$$
\begin{array}{lll}
x^{\prime}(t)=(Q x)(t), & x\left(t_{0}\right)=x_{0} & \text { for } t \geq t_{0} \geq 0 \\
x^{\prime}(t)=(Q x)(t), & x\left(\tau_{0}\right)=y_{0} & \text { for } t \geq \tau_{0} \geq 0
\end{array}
$$

and the perturbed system of (2)

$$
y^{\prime}(t)=(P y)(t), \quad y\left(\tau_{0}\right)=y_{0} \quad \text { for } t \geq \tau_{0},
$$


where $Q, P: E \rightarrow E=C\left(\left[t_{0}, \infty\right], \mathbb{R}^{n}\right)$ is a causal operator and $S \rho=\left[x \in \mathbb{R}^{n}:\|x\|<\rho<\infty\right]$. A special case of (3) is where $(P y)(t)=(Q y)(t)+(R y)(t)$ and $(R y)(t)$ is the perturbation term. We assume the existence and uniqueness of solutions $x(t)$ and $y(t)$ of (1) and (3) through $\left(t_{0}, x_{0}\right)$ and $\left(\tau_{0}, y_{0}\right)$, respectively. We need to have some classes of functions to utilize Lyapunov-like functions for the generalized derivative of Lyapunov functions which has to satisfy suitable conditions as follows:

$$
\begin{aligned}
& E_{A}=\{x \in E: V(s, x(s)) A(s) \leq V(t, x(t)) A(t) \\
&\text { for } \left.t_{0} \leq s \leq t\right\} \\
& E_{1}=\left\{x \in E: V(s, x(s)) \leq V(t, x(t)) \text { for } t_{0} \leq s \leq t\right\},
\end{aligned}
$$

where

(i) $A(t)>0$ is continuously differentiable on $\mathbb{R}_{+}$and $A\left(t_{0}\right)=1$

(ii) $V(t, x) \in C\left[\mathbb{R}_{+} \times \mathbb{R}^{n}, \mathbb{R}_{+}\right]$is a Lyapunov function.

Before we can establish our comparison theorem and Lyapunov stability criteria for initial time difference we need to introduce the following definitions.

Definition 1. The solution $y\left(t, \tau_{0}, y_{0}\right)$ of system (3) through $\left(\tau_{0}, y_{0}\right)$ is said to be initial time difference stable with respect to the solution $\widetilde{x}\left(t, \tau_{0}, x_{0}\right)=x\left(t-\eta, t_{0}, x_{0}\right)$, where $x\left(t, t_{0}, x_{0}\right)$ is any solution of system (1) for $t \geq \tau_{0} \geq 0, t_{0} \in \mathbb{R}_{+}$, and $\eta=\tau_{0}-t_{0}$ if and only if given any $\epsilon>0$ there exist $\delta_{1}=$ $\delta_{1}\left(\epsilon, \tau_{0}\right)>0$ and $\delta_{2}=\delta_{2}\left(\epsilon, \tau_{0}\right)>0$ such that

$$
\left\|y\left(t, \tau_{0}, y_{0}\right)-x\left(t-\eta, t_{0}, x_{0}\right)\right\|<\epsilon
$$

whenever $\left\|y_{0}-x_{0}\right\|<\delta_{1}, \quad\left|\tau_{0}-t_{0}\right|<\delta_{2} \quad$ for $t \geq \tau_{0}$.

If $\delta_{1}, \delta_{2}$ are independent of $\tau_{0}$, then the solution $y\left(t, \tau_{0}, y_{0}\right)$ of system (3) is initial time difference uniformly stable with respect to the solution $x\left(t-\eta, t_{0}, x_{0}\right)$. If the solution $y\left(t, \tau_{0}, y_{0}\right)$ of system (3) through $\left(\tau_{0}, y_{0}\right)$ is initial time difference stable and there exist $\gamma_{1}\left(\tau_{0}\right)>0$ and $\gamma_{2}\left(\tau_{0}\right)>0$ such that

$$
\lim _{t \rightarrow \infty}\left\|y\left(t, \tau_{0}, y_{0}\right)-x\left(t-\eta, t_{0}, x_{0}\right)\right\|=0
$$

for all $y\left(t, \tau_{0}, y_{0}\right)$ and $x\left(t-\eta, t_{0}, x_{0}\right)$ with $\left\|y_{0}-x_{0}\right\|<$ $\gamma_{1}$ and $\left|\tau_{0}-t_{0}\right|<\gamma_{2}$ for $t \geq \tau_{0}$ then it is said to be initial time difference asymptotically stable with respect to the solution $x\left(t-\eta, t_{0}, x_{0}\right)$. It is initial time difference uniformly asymptotically stable with respect to the solution $x\left(t-\eta, t_{0}, x_{0}\right)$ if $\gamma_{1}$ and $\gamma_{2}$ are independent of $\tau_{0}$.

Definition 2. A function $\phi(r)$ is said to belong to the class $\mathscr{K}$ if $\phi \in C\left[(0, \rho), \mathbb{R}_{+}\right], \phi(0)=0$, and $\phi(r)$ is strictly monotone increasing in $r$.
Definition 3. For a real-valued function $V(t, x) \in C\left[\mathbb{R}_{+} \times\right.$ $\left.\mathbb{R}^{n}, \mathbb{R}_{+}\right]$one defines the Dini derivatives as follows:

(a)

$$
\begin{aligned}
& D^{+} V(t, x) \\
& =\lim _{h \rightarrow 0^{+}} \sup \frac{1}{h}[V(t+h, x(t)+h(Q x)(t))-V(t, x(t))] \\
& D_{-} V(t, x) \\
& =\lim _{h \rightarrow 0^{-}} \inf \frac{1}{h}[V(t+h, x(t)+h(Q x)(t))-V(t, x(t))]
\end{aligned}
$$

for $(t, x) \in \mathbb{R}_{+} \times \mathbb{R}^{n}$.

(b) One defines the generalized derivatives (Dini-like derivatives) as follows:

$$
\begin{aligned}
& \begin{aligned}
D_{*}^{+} V(t, y-\tilde{x}) & \\
=\lim _{h \rightarrow 0^{+}} \sup \frac{1}{h}[V(t & +h, y(t)-\widetilde{x(t)} \\
& +h((P y)(t)-(\widetilde{Q x})(t))) \\
- & V(t, y(t)-\widetilde{x}(t))]
\end{aligned} \\
& \begin{aligned}
D_{*}-V(t, y-\tilde{x}) \\
=\lim _{h \rightarrow 0^{-}} \inf \frac{1}{h}[V(t+h, y(t)-\widetilde{x(t)} \\
\quad+h((P y)(t)-(\widetilde{Q \widetilde{x}})(t))) \\
-V(t, y(t)-\widetilde{x}(t))]
\end{aligned}
\end{aligned}
$$

for $(t, x) \in \mathbb{R}_{+} \times \mathbb{R}^{n}$, where $y\left(t, \tau_{0}, y_{0}\right)$ is the solution of system (3) and $\widetilde{x}\left(t, \tau_{0}, x_{0}\right)=x\left(t-\eta, t_{0}, x_{0}\right)$, where $x\left(t, t_{0}, x_{0}\right)$ is any solution of system (1) for $t \geq \tau_{0} \geq 0$, $t_{0} \in \mathbb{R}_{+}$, and $\eta=\tau_{0}-t_{0}$.

(c) Let $V(t, z) \in C\left[\mathbb{R}_{+} \times E, \mathbb{R}_{+}\right]$be any Lyapunov functional. One defines its generalized derivatives as follows:

$$
\begin{aligned}
& D^{+} V(t, y(t)-\tilde{x}(t)) \\
& =\lim _{h \rightarrow 0^{+}} \sup \frac{1}{h}[V(t+h, y(t+h, t, y)-\tilde{x}(t+h, t, \widetilde{x})) \\
& -V(t, y(t)-\tilde{x}(t))] \\
& D_{-} V(t, y(t)-\tilde{x}(t)) \\
& =\lim _{h \rightarrow 0^{-}} \inf \frac{1}{h}[V(t+h, y(t+h, t, y)-\tilde{x}(t+h, t, \tilde{x})) \\
& -V(t, y(t)-\tilde{x}(t))],
\end{aligned}
$$

where $y(t+h, t, y)$ is the solution of IVP (3) through $(t, y)$ and $\widetilde{x}(t+h, t, \widetilde{x})$ is the solution of IVP through $(t, \widetilde{x}), \widetilde{x}(t)=$ $x(t-\eta)$, where $x(t)=x\left(t, t_{0}, x_{0}\right)$ is any solution of the IVP (1), for $t \geq \tau_{0}$. 


\section{Causal Stability and New Notion of ITD Causal Stability}

3.1. Causal Stability. Let $x\left(t, t_{0}, x_{0}\right)$ be any solution of the causal differential system (1) with

$$
x^{\prime}(t)=(Q x)(t), \quad x\left(t_{0}\right)=x_{0} \quad \text { for } t \geq t_{0} \geq 0,
$$

where $Q: E \rightarrow E$ and $S \rho$ is the set $S \rho=\left[x \in \mathbb{R}^{n}:\|x\|<\rho<\right.$ $\infty]$.

Assume that $(Q x)(t)=0$ for $t \geq t_{0} \geq 0$ so that $x \equiv 0$ is a null solution of (1) through $\left(t_{0}, 0\right)$. Now, we can state the well-known definitions concerning the stability of the null solution.

Definition 4. The null solution $x \equiv 0$ of (1) is said to be stable if and only if, for each $\epsilon>0$ and for all $t_{0} \in \mathbb{R}_{+}$, there exists a positive function $\delta=\delta\left(\epsilon, t_{0}\right)$ that is continuous in $t_{0}$ for each $\epsilon$ such that

$$
\left\|x_{0}\right\| \leq \delta \text { implies }\left\|x\left(t, t_{0}, x_{0}\right)\right\|<\epsilon \quad \text { for } t \geq t_{0} .
$$

If $\delta$ is independent of $t_{0}$, then the null solution $x \equiv 0$ of (1) is said to be uniformly stable.

Definition 5. The solution of $x\left(t, t_{0}, y_{0}\right)$ (10) through $\left(\tau_{0}, y_{0}\right)$ is said to be stable with respect to the solution $x\left(t, t_{0}, x_{0}\right)$ of (1) for $t \geq t_{0} \in \mathbb{R}_{+}$if and only if given any $\epsilon>0$ there exists a positive function $\delta=\delta\left(\epsilon, t_{0}\right)$ that is continuous in $t_{0}$ for each $\epsilon$ such that

$$
\begin{array}{r}
\left\|y_{0}-x_{0}\right\| \leq \delta \text { implies }\left\|x\left(t, t_{0}, y_{0}\right)-x\left(t, t_{0}, x_{0}\right)\right\|<\epsilon \\
\text { for } t \geq t_{0} .
\end{array}
$$

If $\delta$ is independent of $t_{0}$, then the solution of system (10) is uniformly stable with respect to the solution $x\left(t, t_{0}, x_{0}\right)$.

We remark that, for the purpose of studying the classical stability of a given solution $x\left(t, t_{0}, y_{0}\right)$ of system (10), it is convenient to make a change of variable. Let $x\left(t, t_{0}, x_{0}\right)$ and $x\left(t, t_{0}, y_{0}\right)$ be the unique solutions of (1) and (10), respectively, and set

$$
z\left(t, t_{0}, y_{0}-x_{0}\right)=x\left(t, t_{0}, y_{0}\right)-x\left(t, t_{0}, x_{0}\right)
$$

for $t \geq t_{0}$. Then

$$
\begin{gathered}
z^{\prime}\left(t, t_{0}, y_{0}-x_{0}\right)=x^{\prime}\left(t, t_{0}, y_{0}\right)-x^{\prime}\left(t, t_{0}, x_{0}\right) \\
z^{\prime}\left(y_{0}-x_{0}\right)(t) \\
=\left(Q z\left(y_{0}-x_{0}\right)+x\left(x_{0}\right)\right)(t)-\left(Q x\left(x_{0}\right)\right)(t) \\
z^{\prime}\left(y_{0}-x_{0}\right)(t)=\left(\widetilde{Q} z\left(y_{0}-x_{0}\right)\right)(t) .
\end{gathered}
$$

It is easy to observe that $z\left(t, t_{0}, y_{0}-x_{0}\right) \equiv 0$ is a solution of the transformed system if $y_{0}-x_{0}=0$ which implies $(Q 0)(t)=0$ for $t \geq t_{0} \geq 0$. Since $z^{\prime}(t)=0$ and $z\left(t, t_{0}, 0\right)=0$ is the null solution, the solution of $x\left(t, t_{0}, x_{0}\right)$ (1) corresponds to the identically null solution of $z^{\prime}(t)=\left(\widetilde{Q} z\left(y_{0}-x_{0}\right)\right)(t)$ where $\left(\widetilde{Q} z\left(y_{0}-x_{0}\right)\right)(t)=\left(Q z\left(y_{0}-x_{0}\right)+x\left(x_{0}\right)\right)(t)-$ $\left(\mathrm{Q} x\left(x_{0}\right)\right)(t)$. Hence, we can assume, without loss of generality, that $x\left(t, t_{0}, x_{0}\right) \equiv 0$ is the null solution of system (10) and we can limit our study of stability to that of the null solution [5]. However, it is not possible to do a similar transformation for initial time difference stability analysis.

3.2. New Notion of ITD Causal Stability. Let $x\left(t, \tau_{0}, y_{0}\right)$ be a solution of (2) and $\tilde{x}\left(t, \tau_{0}, x_{0}\right)=x\left(t-\eta, t_{0}, x_{0}\right)$ where $x\left(t, t_{0}, x_{0}\right)$ is any solution of system (1) for $t \geq \tau_{0} \geq 0$. Let us make a transformation similar to that in (15). Set $z\left(t, \tau_{0}, y_{0}-\right.$ $\left.x_{0}\right)=x\left(t, \tau_{0}, y_{0}\right)-x\left(t-\eta, t_{0}, x_{0}\right)$ for $t \geq \tau_{0}$. Then

$$
\begin{gathered}
z^{\prime}\left(t, \tau_{0}, y_{0}-x_{0}\right)=x^{\prime}\left(t, \tau_{0}, y_{0}\right)-x^{\prime}\left(t-\eta, t_{0}, x_{0}\right) \\
z^{\prime}\left(y_{0}-x_{0}\right)(t)=\left(\widetilde{Q} z\left(y_{0}-x_{0}\right)\right)(t ; \eta) .
\end{gathered}
$$

One can observe that even if $y_{0}=x_{0}, z\left(t, \tau_{0}, 0\right)$ is not zero and is not the null solution of the transformed system and the solution $x\left(t-\eta, t_{0}, x_{0}\right)$ does not correspond to the identically zero solution of $z^{\prime}(t)=\left(\widetilde{Q} z\left(y_{0}-x_{0}\right)\right)(t ; \eta)$. Consequently, stability properties of null solution cannot be used in order to find ITD stability properties using this approach.

\section{Comparison Theorems via Lyapunov Functions with Initial Time Difference}

In our earlier work and in the work of others $[1,2,5]$, the differences between the classical notion of stability and ITD stability did not allow the use of the behavior of the null solution in our ITD stability analysis. The main result presented in this section resolves those difficulties with a new approach that allows the use of the stability of the null solution of the comparison system to predict the stability properties of $y\left(t, \tau_{0}, y_{0}\right)$ the solution of (3) with respect to $\widetilde{x}(t)=x\left(t-\eta, t_{0}, x_{0}\right)$ where $x\left(t, t_{0}, x_{0}\right)$ is any solution of system (1).

Theorem 6. Assume that

(i) $V(t, z) \in C\left[\mathbb{R}_{+} \times \mathbb{R}^{n}, \mathbb{R}_{+}\right]$and $V(t, z)$ is locally Lipschitzian in $z$;

(ii) for $t \geq \tau_{0}$ and $x \in E_{1}$,

$$
D_{*}^{+} V(t, y-\tilde{x}) \leq G(t, V(t, y(t)-\tilde{x}(t))),
$$

where

$$
\begin{aligned}
& D_{*}^{+} V(t, y(t)-\tilde{x}(t)) \\
&=\lim _{h \rightarrow 0^{+}} \sup \frac{1}{h}[V(t+h, y(t)-\tilde{x}(t) \\
&+h((P y)(t)-(\widetilde{Q} \tilde{x})(t))) \\
&-V(t, y(t)-\tilde{x}(t))]
\end{aligned}
$$

with $(t, \tilde{x}),(t, y) \in \mathbb{R}_{+} \times \mathbb{R}^{n}$ and $G \in C\left[\mathbb{R}_{+} \times \mathbb{R}_{+}, \mathbb{R}_{+}\right]$; 
(iii) $r(t)=r\left(t, \tau_{0}, u_{0}\right)$ is the maximal solution of the scalar differential equation

$$
u^{\prime}=G(t, u), u\left(\tau_{0}\right)=u_{0} \geq 0 \quad \text { for } t \geq \tau_{0}
$$

existing on $\left[\tau_{0}, \infty\right)$.

Then, if $\tilde{x}(t)=x\left(t-\eta, t_{0}, x_{0}\right), \eta=\tau_{0}-t_{0}$, where $x\left(t, t_{0}, x_{0}\right)$ is any solution of the causal differential system (1) and $y(t)=y\left(t, \tau_{0}, y_{0}\right)$ is any solution of causal differential system (3) existing on $\left[\tau_{0}, \infty\right)$ such that $V\left(\tau_{0}, y_{0}-x_{0}\right) \leq u_{0}$ implies

$$
V(t, y(t)-\tilde{x}(t)) \leq r(t) \quad \text { for } t \geq \tau_{0}
$$

Proof. Let $\tilde{x}(t)=x\left(t-\eta, t_{0}, x_{0}\right), \eta=\tau_{0}-t_{0}$, and let $x\left(t, t_{0}, x_{0}\right)$ be any solution of the causal differential system (1) and let $y(t)=y\left(t, \tau_{0}, y_{0}\right)$ be any solution of causal differential system (3) for $t \geq \tau_{0}$. Define

$$
m(t)=V(t, y(t)-\tilde{x}(t)) \quad \text { for } t \geq \tau_{0}
$$

so that $m\left(\tau_{0}\right)=V\left(\tau_{0}, y\left(\tau_{0}\right)-\tilde{x}\left(\tau_{0}\right)\right) \leq u_{0}$. For some sufficiently small $\varepsilon>0$, consider the differential equation

$$
u^{\prime}=G(t, u)+\varepsilon, \quad u\left(\tau_{0}\right)=u_{0}+\varepsilon \quad \text { for } t \geq \tau_{0}
$$

whose solutions $u(t, \varepsilon)=u\left(t, \tau_{0}, u_{0}, \varepsilon\right)$ exist as far as $r(t)$. In order to prove the conclusion of the theorem, we need to show that

$$
m(t)=V(t, y(t)-\tilde{x}(t))<u(t, \varepsilon) \quad \text { for } t \geq \tau_{0} .
$$

If this is not true, then there exists a $t_{1} \geq \tau_{0}$ such that

$$
\begin{gathered}
m(t)<u(t, \varepsilon) \quad \text { for } t_{1}>t \geq \tau_{0}, \\
m\left(t_{1}\right)=u\left(t_{1}, \varepsilon\right) .
\end{gathered}
$$

It then follows that

$$
D^{+} m\left(t_{1}\right) \geq u^{\prime}\left(t_{1}, \varepsilon\right)=G\left(t_{1}, u\left(t_{1}, \varepsilon\right)\right)+\varepsilon .
$$

By using the assumption on $G$ and $G(t, u) \geq 0$, the solutions $u(t, \varepsilon)$ are nondecreasing in $t$. Since $m(t)=V(t, y(t)-\tilde{x}(t))$ for $t \geq \tau_{0}$, we get

$$
V(s, y(s)-\tilde{x}(s)) \leq u\left(t_{1}, \varepsilon\right) \quad \text { for } t_{1} \geq s \geq \tau_{0} .
$$

Consequently, $(y-\tilde{x})(t) \in E_{1}$.
The standard computation for small enough $h>0$ implies

$$
\begin{aligned}
m & (t+h)-m(t) \\
= & V(t+h, y(t+h)-\tilde{x}(t+h))-V(t, y(t)-\tilde{x}(t)) \\
\leq & V(t+h, y(t+h)-\tilde{x}(t+h)) \\
& -V(t+h, y(t)-\tilde{x}(t)+h((P y)(t)-(\widetilde{Q} \tilde{x})(t))) \\
& +V(t+h, y(t)-\tilde{x}(t)+h((P y)(t)-(\widetilde{Q} \tilde{x})(t))) \\
& -V(t, y(t)-\tilde{x}(t)) \\
\leq & L \|[y(t+h)-y(t)]-[\tilde{x}(t+h)-\tilde{x}(t)] \\
& \quad-h((P y)(t)-(\widetilde{Q} \tilde{x})(t)) \| \\
& +V(t+h, y(t)-\tilde{x}(t)+h((P y)(t)-(\widetilde{Q} \tilde{x})(t))) \\
& -V(t, y(t)-\tilde{x}(t)) .
\end{aligned}
$$

Since $V(t, z)$ is locally Lipschitzian in $z$ and $L>0$ is the Lipschitzian constant, we obtain

$$
\begin{aligned}
& m(t+h)-m(t) \\
& \leq L\left[\epsilon_{1}(h)-\epsilon_{2}(h)\right] \\
& \quad+V(t+h, y(t)-\widetilde{x}(t)+h((P y)(t)-(\widetilde{Q} \tilde{x})(t))) \\
& \quad-V(t, y(t)-\tilde{x}(t)),
\end{aligned}
$$

where $\epsilon_{1}$ and $\epsilon_{2}$ are error terms. This shows that

$$
\begin{aligned}
& D^{+} m(t) \\
& =\lim _{h \rightarrow 0^{+}} \sup \frac{1}{h} L\left[\epsilon_{1}(h)-\epsilon_{2}(h)\right] \\
& \quad+\lim _{h \rightarrow 0^{+}} \sup \frac{1}{h}[V(t+h, y(t)-\tilde{x}(t) \\
& \quad+h((P y)(t)-(\widetilde{Q} \tilde{x})(t))) \\
& \quad-V(t, y(t)-\tilde{x}(t))] \\
& \leq G(t, V(t, y(t)-\tilde{x}(t))) \\
& =G(t, m(t)) \quad \text { for } \tau_{0} \leq t \leq t_{1}<\infty
\end{aligned}
$$

since $\left(\epsilon_{1}(h)-\epsilon_{2}(h)\right) / h \rightarrow 0$ as $h \rightarrow 0$. Hence, at $t=t_{1}$, we have

$$
\begin{aligned}
D^{+} m\left(t_{1}\right) & \leq D_{*}^{+} V\left(t_{1}, y\left(t_{1}\right)-\tilde{x}\left(t_{1}\right)\right) \\
& \leq G\left(t_{1}, V\left(t, y\left(t_{1}\right)-\tilde{x}\left(t_{1}\right)\right)\right)=G\left(t_{1}, m\left(t_{1}\right)\right) \\
& <G\left(t_{1}, u\left(t_{1}, \varepsilon\right)\right)+\varepsilon
\end{aligned}
$$

which contradicts (27). Hence $m(t)<u(t, \varepsilon)$, which yields the desired estimate as $\varepsilon \rightarrow 0$ :

$$
m(t)=V(t, y(t)-\tilde{x}(t)) \leq r\left(t, \tau_{0}, u_{0}\right) \quad \text { for } t \geq \tau_{0} .
$$

Therefore these complete the proof. 
Corollary 7. Let $V$ satisfy the conditions of Theorem 6 with $G(t, u) \equiv 0$ and $x(t) \in E_{1}$. Then

$$
V(t, y(t)-\tilde{x}(t)) \leq V\left(\tau_{0}, y_{0}-x_{0}\right) \quad \text { for } t \geq \tau_{0},
$$

where $x(t)$ and $y(t)$ are any solutions of the initial value problems (1) and (2), respectively. Equivalently we have

$$
\begin{array}{r}
V\left(t_{2}, y\left(t_{2}\right)-\tilde{x}\left(t_{2}\right)\right) \leq V\left(t_{1}, y\left(t_{1}\right)-\tilde{x}\left(t_{1}\right)\right) \\
\text { for } \tau_{0} \leq t_{1} \leq t_{2}<\infty .
\end{array}
$$

Theorem 8. Assume that

(i) $V(t, z) \in C\left[\mathbb{R}_{+} \times \mathbb{R}^{n}, \mathbb{R}_{+}\right]$and $V(t, z)$ is locally Lipschitzian in $z$

(ii) fort $\geq \tau_{0}$ and $x \in E_{A}$,

$$
\begin{aligned}
& A(t) D_{*}^{+} V(t, y(t)-\tilde{x}(t))+V(t, y(t)-\tilde{x}(t)) A^{\prime}(t) \\
& \quad \leq G(t, V(t, y(t)-\tilde{x}(t)) A(t)),
\end{aligned}
$$

where

$$
\begin{aligned}
D_{*}^{+} V(t, y(t)-\tilde{x}(t)) & \\
=\lim _{h \rightarrow 0^{+}} \sup \frac{1}{h}[V( & +h, y(t)-\widetilde{x}(t) \\
+ & h((P y)(t)-(\widetilde{Q} \tilde{x})(t))) \\
- & V(t, y(t)-\widetilde{x}(t))]
\end{aligned}
$$

with $(t, \tilde{x}),(t, y) \in \mathbb{R}_{+} \times \mathbb{R}^{n}$ and $G \in C\left[\mathbb{R}_{+} \times \mathbb{R}_{+}, \mathbb{R}_{+}\right]$;

(iii) $r(t)=r\left(t, \tau_{0}, u_{0}\right)$ is the maximal solution of the scalar differential equation

$$
u^{\prime}=G(t, u), \quad u\left(\tau_{0}\right)=u_{0} \geq 0 \quad \text { for } t \geq \tau_{0}
$$

existing on $\left[\tau_{0}, \infty\right)$.

Then, if $\widetilde{x}(t)=x\left(t-\eta, t_{0}, x_{0}\right), \eta=\tau_{0}-t_{0}$, where $x(t)$ is any solution of the system (1) and $y(t)=y\left(t, \tau_{0}, y_{0}\right)$ is any solution of the system (3) existing on $\left[\tau_{0}, \infty\right)$ such that $A\left(\tau_{0}\right) V\left(\tau_{0}, y_{0}-\right.$ $\left.x_{0}\right) \leq u_{0}$ implies

$$
V(t, y(t)-\tilde{x}(t)) A(t) \leq r(t) \quad \text { for } t \geq \tau_{0} .
$$

Proof. Define $L(t, y(t)-\widetilde{x}(t))=V(t, y(t)-\widetilde{x}(t)) A(t)$. Let $t \geq$ $\tau_{0}$ and $x(t) \in E_{A}$. Then it is easy to see that

$$
\begin{aligned}
& D^{+} L(t, y(t)-\tilde{x}(t)) \\
& \leq A(t) D_{*}^{+} V(t, y(t)-\tilde{x}(t))+V(t, y(t)-\tilde{x}(t)) A^{\prime}(t) \\
& \leq G(t, V(t, y(t)-\tilde{x}(t)) A(t)) \\
& =G(t, L(t, y(t)-\tilde{x}(t))) .
\end{aligned}
$$

Then, by the application of Theorem 6 , it follows that

$$
\begin{array}{r}
V(t, y(t)-\tilde{x}(t)) A(t)=L(t, y(t)-\tilde{x}(t)) \leq r(t) \\
\text { for } t \geq \tau_{0} .
\end{array}
$$

\section{Initial Time Difference Stability of Causal Differential Systems}

5.1. Initial Time Difference Stability Criteria via Lyapunov Functions. We will give sufficient conditions for the stability of the unperturbed systems $x(t)=x\left(t, t_{0}, x_{0}\right)$ of (1) in terms of Lyapunov functions and we assume that the solutions of causal differential systems of (1) exist and are unique for $t \geq$ $\tau_{0}$.

Theorem 9. Assume the following.

(i) Let $V(t, z) \in C\left[\mathbb{R}_{+} \times S(\rho), \mathbb{R}_{+}\right]$and let $V(t, z)$ be locally Lipschitzian in $z$;

(ii) for $t \geq \tau_{0}$ and $x \in E_{1}$,

$$
D_{*}^{+} V(t, y-\tilde{x}) \leq 0,
$$

where

$$
\begin{aligned}
D_{*}^{+} V(t, y(t)-\tilde{x}(t)) & \\
=\lim _{h \rightarrow 0^{+}} \sup \frac{1}{h}[V(t+h, y(t)-\tilde{x}(t) & +h((P y)(t)-(\widetilde{\mathrm{Q}} \tilde{x})(t))) \\
& -V(t, y(t)-\tilde{x}(t))]
\end{aligned}
$$

with $(t, \widetilde{x}),(t, y) \in \mathbb{R}_{+} \times S(\rho)$;

(iii) let $V(t, z)$ be positive definite and decrescent on $\mathbb{R}_{+} \times$ $S(\rho): V(t, z)$ satisfies

$$
\begin{array}{r}
a(\|z\|) \leq V(t, z) \leq b(\|z\|), \quad(t, z) \in \mathbb{R}_{+} \times S(\rho), \\
a, b \in \mathscr{K} .
\end{array}
$$

Then the solution of perturbed causal differential system $y\left(t, \tau_{0}, y_{0}\right)$ of (3) is initial time difference stable with respect to the solution of unperturbed causal differential system $\tilde{x}\left(t, \tau_{0}, x_{0}\right)=x\left(t-\eta, t_{0}, x_{0}\right)$, where $x\left(t, t_{0}, x_{0}\right)$ is any solution of system (1), for $t \geq \tau_{0}$.

Proof. Let $\varepsilon>0$ and $t \geq \tau_{0}$ be given. Choose $\delta_{1}\left(\epsilon, \tau_{0}\right)>0$ and $\delta_{2}\left(\epsilon, \tau_{0}\right)>0$ such that $b(\delta)<a(\varepsilon)$ where $\delta\left(\epsilon, \tau_{0}\right)=$ $\max \left[\delta_{1}\left(\epsilon, \tau_{0}\right), \delta_{2}\left(\epsilon, \tau_{0}\right)\right]>0$. Then, we claim that, with this $\delta$, stability of the solution $x\left(t, t_{0}, x_{0}\right)$ of unperturbed causal differential system of (1) follows for $t \geq t_{0}$. If this is not true, then there would exist solutions $y\left(t, \tau_{0}, y_{0}\right)$ of $(3), \widetilde{x}\left(t, \tau_{0}, x_{0}\right)$ for $t \geq \tau_{0}$, and $t_{2}>t_{1}>\tau_{0}$ satisfying

$$
\begin{aligned}
& \left\|y\left(t_{1}\right)-\tilde{x}\left(t_{1}\right)\right\|=\delta, \quad\left\|y\left(t_{2}\right)-\tilde{x}\left(t_{2}\right)\right\|=\varepsilon, \\
& \delta \leq\|y(t)-\tilde{x}(t)\| \leq \varepsilon \quad \text { for } t_{1} \leq t \leq t_{2} .
\end{aligned}
$$

Then, by using assumption (ii) and Corollary 7, we get the estimate

$$
\begin{array}{r}
V\left(t_{2}, y\left(t_{2}\right)-\tilde{x}\left(t_{2}\right)\right) \leq V\left(t_{1}, y\left(t_{1}\right)-\tilde{x}\left(t_{1}\right)\right) \\
\text { for } t_{1} \leq t \leq t_{2}<\infty .
\end{array}
$$

These complete the proof. 
Therefore, using (45) and assumption (iii), together with the choice of $\delta$, implies

$$
\begin{aligned}
a(\varepsilon) & =a\left(\left\|y\left(t_{2}\right)-\tilde{x}\left(t_{2}\right)\right\|\right) \leq V\left(t_{2}, y\left(t_{2}\right)-\tilde{x}\left(t_{2}\right)\right) \\
& \leq V\left(t_{1}, y\left(t_{1}\right)-\tilde{x}\left(t_{1}\right)\right) \leq b\left(\left\|y\left(t_{1}\right)-\tilde{x}\left(t_{1}\right)\right\|\right) \\
& =b(\delta)<a(\varepsilon)
\end{aligned}
$$

which is a contradiction. Hence the solution $x\left(t, t_{0}, x_{0}\right)$ of unperturbed causal system of (1) is stable for $t \geq t_{0}$.

If $\delta_{1}=\delta_{1}\left(\epsilon, \tau_{0}\right)>0$ and $\delta_{2}=\delta_{2}\left(\epsilon, \tau_{0}\right)>0$ are independent of $\tau_{0}$, then the solution $x\left(t, t_{0}, x_{0}\right)$ of unperturbed causal system of (1) is uniformly stable for $t \geq t_{0}$.

Theorem 10. Assume the following.

(i) Let $V(t, z) \in C\left[\mathbb{R}_{+} \times S(\rho), \mathbb{R}_{+}\right]$and let $V(t, z)$ be locally Lipschitzian in $z$;

(ii) for $t \geq \tau_{0}$ and $x \in E_{A}$,

$D_{*}^{+} V(t, y(t)-\tilde{x}(t)) A(t)+V(t, y(t)-\tilde{x}(t)) A^{\prime}(t) \leq 0$,

where $A(t)$ is continuously differentiable for $t \geq \tau_{0}$ with $A\left(\tau_{0}\right)=1, A(t) \geq 1$, and $A(t) \rightarrow \infty$ as $t \rightarrow \infty$,

$D_{*}^{+} V(t, y(t)-\tilde{x}(t))$

$=\lim _{h \rightarrow 0^{+}} \sup \frac{1}{h}[V(t+h, y(t)-\tilde{x}(t)$

$$
+h((P y)(t)-(\widetilde{Q} \tilde{x})(t)))
$$$$
-V(t, y(t)-\tilde{x}(t))]
$$

with $(t, \tilde{x}),(t, y) \in \mathbb{R}_{+} \times S(\rho) ;$

(iii) let $V(t, z)$ be positive definite and decrescent on $\mathbb{R}_{+} \times$ $S(\rho): V(t, z)$ satisfies

$$
\begin{array}{r}
a(\|z\|) \leq V(t, z) \leq b(\|z\|), \quad(t, z) \in \mathbb{R}_{+} \times S(\rho), \\
a, b \in \mathscr{K} .
\end{array}
$$

Then the solution of perturbed causal differential system $y\left(t, \tau_{0}, y_{0}\right)$ of (3) is initial time difference asymptotically stable with respect to the solution of unperturbed causal differential system $\tilde{x}\left(t, \tau_{0}, x_{0}\right)=x\left(t-\eta, t_{0}, x_{0}\right)$, where $x\left(t, t_{0}, x_{0}\right)$ is any solution of system (1), for $t \geq \tau_{0}$.

Proof. By applying Theorem 8, we get

$$
V(t, y(t)-\tilde{x}(t)) A(t) \leq V\left(\tau_{0}, y_{0}-x_{0}\right) \quad \text { for } t \geq \tau_{0} .
$$

By Theorem 9, we have the solution $x\left(t, t_{0}, x_{0}\right)$ of unperturbed causal system of (1) which is stable for $t \geq t_{0}$. We only have to prove quasiasymptotic stability. In order to do that, let $\varepsilon=\rho$ so that $\delta_{0}=\delta\left(\rho, \tau_{0}\right)$ where $\delta\left(\rho, \tau_{0}\right)=$ $\max \left[\delta_{1}\left(\rho, \tau_{0}\right), \delta_{2}\left(\rho, \tau_{0}\right)\right]>0$. Choose $\left\|y_{0}-x_{0}\right\|<\delta_{0}$ and $\left|\tau_{0}-t_{0}\right|<\delta_{2}$. Then, in view of (ii), (iii), and (51), it follows that, given any $\varepsilon>0$ and $\tau_{0} \geq 0$, there exists a $T=T\left(\varepsilon, \tau_{0}\right)>0$ satisfying

$$
\begin{aligned}
& a(\|y(t)-\tilde{x}(t)\|) \\
& \leq V(t, y(t)-\tilde{x}(t)) \leq V\left(\tau_{0}, y_{0}-x_{0}\right) A^{-1}(t) \\
& \leq b\left(\left\|y_{0}-x_{0}\right\|\right) A^{-1}(t)<b\left(\delta_{0}\right) A^{-1}(t)<\varepsilon
\end{aligned}
$$

for $t \geq \tau_{0}+T$ since $A^{-1}(t)$ does exist and $A^{-1}(t)=1 / A(t) \rightarrow$ 0 as $t \rightarrow \infty$. Hence the solution $x\left(t, t_{0}, x_{0}\right)$ of unperturbed causal system of (1) is quasiasymptotically stable for $t \geq t_{0}$. Therefore the solution of perturbed causal differential system $y\left(t, \tau_{0}, y_{0}\right)$ of (3) is initial time difference asymptotically stable with respect to the solution of unperturbed causal differential system $\tilde{x}\left(t, \tau_{0}, x_{0}\right)=x\left(t-\eta, t_{0}, x_{0}\right)$ or the solution $x\left(t, t_{0}, x_{0}\right)$ of unperturbed causal system of (1) is asymptotically stable for $t \geq t_{0}$.

If $\delta_{1}=\delta_{1}\left(\epsilon, \tau_{0}\right)>0, \delta_{2}=\delta_{2}\left(\epsilon, \tau_{0}\right)>0$ and $T=T\left(\varepsilon, \tau_{0}\right)>$ 0 are independent of $\tau_{0}$, then the solution $x\left(t, t_{0}, x_{0}\right)$ of unperturbed causal system of (1) is uniformly asymptotically stable for $t \geq t_{0}$.

5.2. Initial Time Difference Stability Criteria via Lyapunov Functionals. In this section, we employ Lyapunov functional for discussing initial time difference stability theory in the context of causal differential equations.

We will give a result parallel to the original Lyapunov second method on uniformly asymptotic stability with initial time difference.

Theorem 11. Assume the following.

(i) Let $V(t, z) \in C\left[\mathbb{R}_{+} \times E, \mathbb{R}_{+}\right]$and $D^{+} V(t, y(t)-\tilde{x}(t)) \leq$ $-c(\|y(t)-\tilde{x}(t)\|), c \in \mathscr{K}$;

(ii) let $V(t, z)$ be positive definite and decrescent on $\mathbb{R}_{+} \times E$ : $V(t, z)$ satisfies

$$
\begin{array}{r}
a(\|z\|) \leq V(t, z) \leq b(\|z\|), \quad(t, z) \in \mathbb{R}_{+} \times E, \\
a, b \in \mathscr{K} .
\end{array}
$$

Then the solution of perturbed causal differential system $y\left(t, \tau_{0}, y_{0}\right)$ of (3) is initial time difference uniformly asymptotically stable with respect to the solution of unperturbed causal differential system $\tilde{x}\left(t, \tau_{0}, x_{0}\right)=x\left(t-\eta, t_{0}, x_{0}\right)$, where $x\left(t, t_{0}, x_{0}\right)$ is any solution of system (1), for $t \geq \tau_{0}$.

Proof. Let $\varepsilon>0$ and $t \geq \tau_{0}$ be given. Choose $\delta_{1}\left(\epsilon, \tau_{0}\right)=$ $\delta_{1}(\epsilon)>0$ and $\delta_{2}\left(\epsilon, \tau_{0}\right)=\delta_{2}(\epsilon)>0$ such that

$$
b(\delta)<a(\varepsilon)
$$

where $\delta(\epsilon)=\min \left[\delta_{1}(\epsilon), \delta_{2}(\epsilon)\right]>0$. Then, we have that, with this $\delta$ and $\varepsilon$, stability of the solution $x\left(t, t_{0}, x_{0}\right)$ of unperturbed causal differential system of (1) follows for $t \geq t_{0}$. If this is 
not true, then there would exist solutions $y\left(t, \tau_{0}, y_{0}\right)$ of (3), $\widetilde{x}\left(t, \tau_{0}, x_{0}\right)$ for $t \geq \tau_{0}$, and $t_{1}>\tau_{0}$ satisfying

$$
\begin{gathered}
\left\|y\left(t_{1}, \tau_{0}, y_{0}\right)-\tilde{x}\left(t_{1}, \tau_{0}, x_{0}\right)\right\|=\varepsilon, \\
\left\|y\left(t, \tau_{0}, y_{0}\right)-\tilde{x}\left(t, \tau_{0}, x_{0}\right)\right\| \leq \varepsilon \\
\text { for } \tau_{0} \leq t \leq t_{1} .
\end{gathered}
$$

Then, by using assumption (ii) and Corollary 7, we get the estimate

$$
\begin{array}{r}
V\left(t, y\left(t, \tau_{0}, y_{0}\right)-\tilde{x}\left(t, \tau_{0}, x_{0}\right)\right) \leq V\left(\tau_{0}, y_{0}-x_{0}\right) \\
\text { for } \tau_{0} \leq t \leq t_{1}<\infty .
\end{array}
$$

Therefore, using (54), (55), (56), and assumption (ii), together with the choice of $\delta$, yields

$$
\begin{aligned}
a(\varepsilon) & =a\left(\left\|y\left(t_{1}, \tau_{0}, y_{0}\right)-\tilde{x}\left(t_{1}, \tau_{0}, x_{0}\right)\right\|\right) \\
& \leq V\left(t_{1}, y\left(t_{1}, \tau_{0}, y_{0}\right)-\tilde{x}\left(t_{1}, \tau_{0}, x_{0}\right)\right) \\
& \leq V\left(\tau_{0}, y_{0}-x_{0}\right) \leq b\left(\left\|y_{0}-x_{0}\right\|\right)=b(\delta)<a(\varepsilon)
\end{aligned}
$$

which is a contradiction. Hence the solution $x\left(t, t_{0}, x_{0}\right)$ of unperturbed causal system of (1) is uniformly stable for $t \geq t_{0}$ as follows.

To prove uniformly asymptotic stability, set $\varepsilon=\rho$ and designate $\delta_{0}=\delta(\rho)$ so that

$$
\begin{array}{r}
\left\|y_{0}-x_{0}\right\|<\delta_{0}, \quad\left|\tau_{0}-t_{0}\right|<\delta_{0} \\
\text { implies }\left\|y\left(t, \tau_{0}, y_{0}\right)-x\left(t-\eta, t_{0}, x_{0}\right)\right\|<\rho \\
\text { for } t \geq \tau_{0} .
\end{array}
$$

In view of uniform stability, it is easy to show that there exists a $t^{*}, \tau_{0}<t^{*}<\tau_{0}+T$, where $T=1+b\left(\delta_{0}\right) / c(\delta)$ and $\left\|y_{0}-x_{0}\right\|<$ $\delta_{0}$ and $\left|\tau_{0}-t_{0}\right|<\delta_{0}$ and $\left\|y\left(t^{*}, \tau_{0}, y_{0}\right)-\widetilde{x}\left(t^{*}, \tau_{0}, x_{0}\right)\right\|<\delta(\varepsilon)$. Here $\delta=\delta(\varepsilon)$ corresponds to $\varepsilon>0$ for uniform stability. If it is not, let $\delta \leq\left\|y\left(t, \tau_{0}, y_{0}\right)-\widetilde{x}\left(t, \tau_{0}, x_{0}\right)\right\|, t \in\left[\tau_{0}, \tau_{0}+T\right]$. Then, by assumption (i), we get

$$
\begin{aligned}
& V\left(t, y\left(t, \tau_{0}, y_{0}\right)-\tilde{x}\left(t, \tau_{0}, x_{0}\right)\right) \\
& \leq V\left(\tau_{0}, y_{0}-x_{0}\right)-\int_{\tau_{0}}^{t} c\left(y\left(s, \tau_{0}, y_{0}\right)-\tilde{x}\left(s, \tau_{0}, x_{0}\right)\right) d s
\end{aligned}
$$

for $\left[\tau_{0}, \tau_{0}+T\right]$. As a result of this

$$
\begin{aligned}
0 & \leq V\left(\tau_{0}+T, y\left(\tau_{0}+T, \tau_{0}, y_{0}\right)-\tilde{x}\left(\tau_{0}+T, \tau_{0}, x_{0}\right)\right) \\
& \leq V\left(\tau_{0}, y_{0}-x_{0}\right)-\int_{\tau_{0}}^{\tau_{0}+T} c\left(y\left(s, \tau_{0}, y_{0}\right)-\tilde{x}\left(s, \tau_{0}, x_{0}\right)\right) d s \\
& \leq b\left(\delta_{0}\right)-c(\delta) T<0
\end{aligned}
$$

by the definition of $T$. This contradiction shows that there exists a $t^{*}>\tau_{0}$ such that $\left\|y\left(t^{*}, \tau_{0}, y_{0}\right)-x\left(t^{*}-\eta, t_{0}, x_{0}\right)\right\|<\delta$. This implies, by stability, that

$$
\begin{aligned}
& \left\|y_{0}-x_{0}\right\|<\delta, \quad\left|\tau_{0}-t_{0}\right|<\delta, \\
& \left\|y\left(t, \tau_{0}, y_{0}\right)-x\left(t-\eta, t_{0}, x_{0}\right)\right\|<\varepsilon
\end{aligned}
$$

$$
\text { for } t \geq \tau_{0}+T \text {. }
$$

This completes the proof.

\section{Conflict of Interests}

The authors declare that there is no conflict of interests regarding the publication of this paper.

\section{Acknowledgments}

This work has been supported by The Yıldız Technical University and Yeditepe University Department of Mathematics and The Scientific and Technological Research Council of Turkey. The authors also would like to thank V. Lakshmikantham and the referees for their insightful comments and detailed suggestions which improved the quality of the paper.

\section{References}

[1] V. Lakshmikantham, S. Leela, Z. Drici, and F. A. McRae, Theory of Causal Differential Equations, vol. 5 of Atlantis Studies in Mathematics for Engineering and Science, 2010.

[2] V. Lakshmikantham and S. Leela, Differential and Integral Inequalities, vol. 1, Academic Press, New York, NY, USA, 1969.

[3] V. Lakshmikantham and A. S. Vatsala, "Differential inequalities with initial time difference and applications," Journal of Inequalities and Applications, vol. 3, no. 3, pp. 233-244, 1999.

[4] M. D. Shaw and C. Yakar, "Generalized variation of parameters with initial time difference and a comparison result in term Lyapunov-like functions," International Journal of Nonlinear Differential Equations, Theory Methods and Applications, vol. 5, pp. 86-108, 1999.

[5] F. Brauer and J. Nohel, The Qualitative Theory of Ordinary Differential Equations, W.A. Benjamin, New York, NY, USA, 1969. 


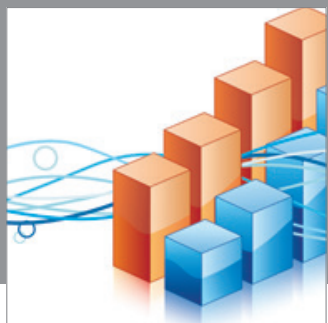

Advances in

Operations Research

mansans

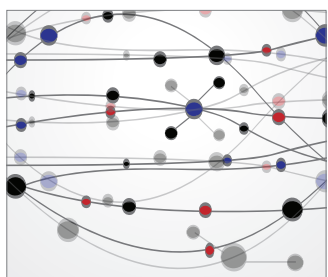

The Scientific World Journal
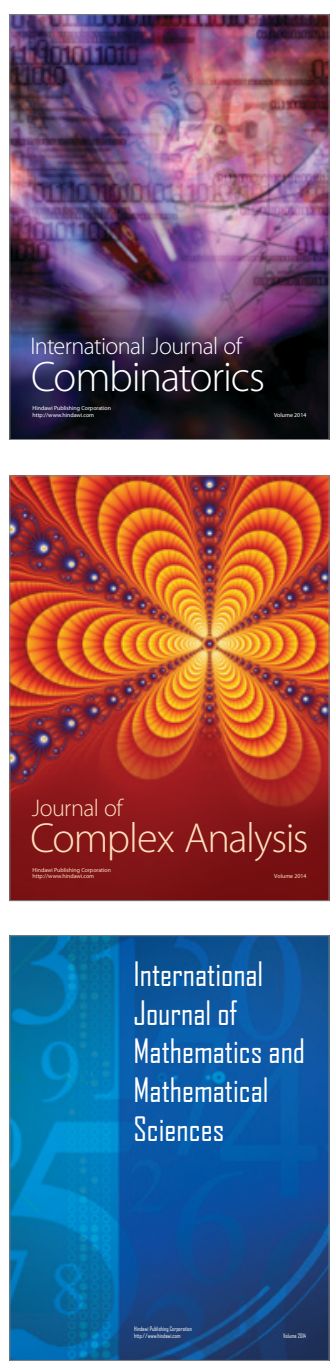
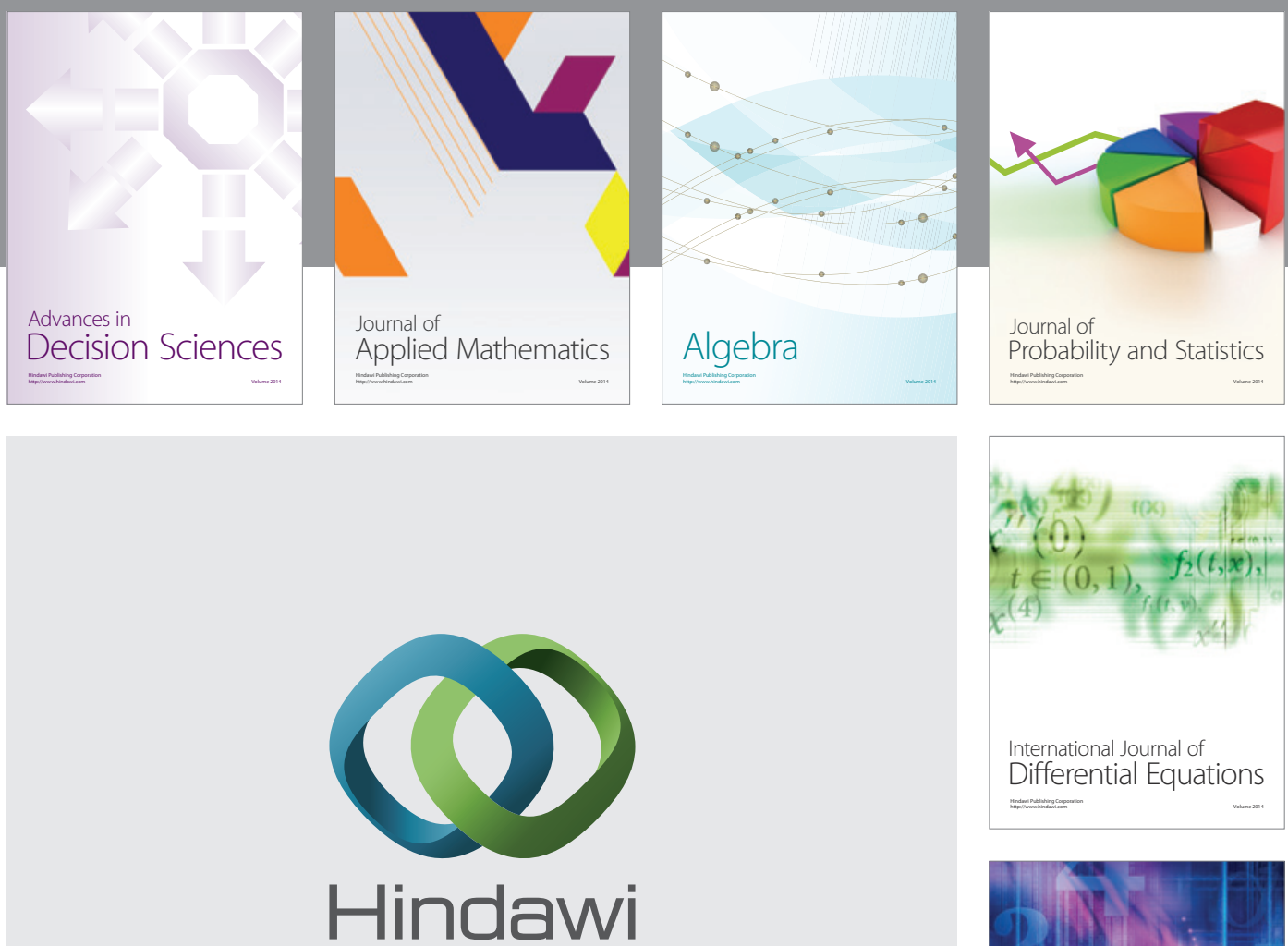

Submit your manuscripts at http://www.hindawi.com
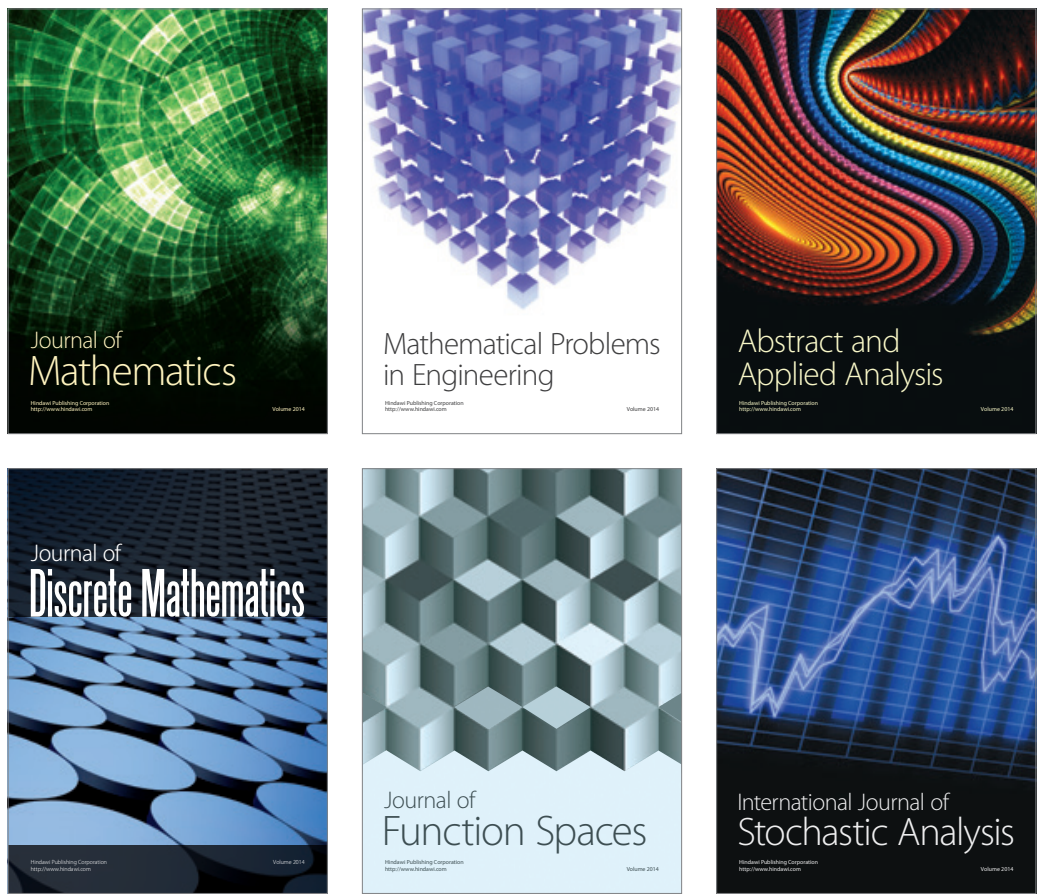

Journal of

Function Spaces

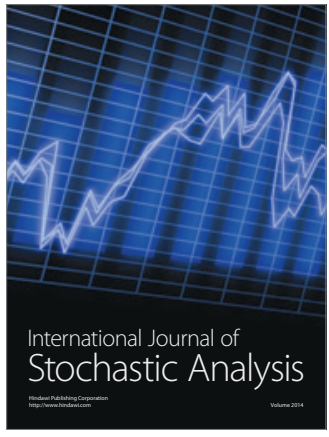

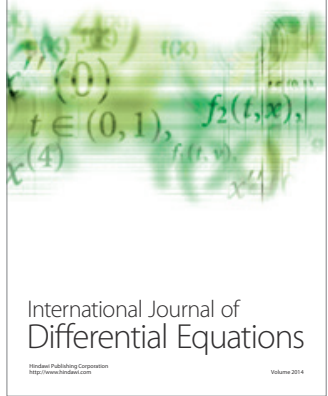
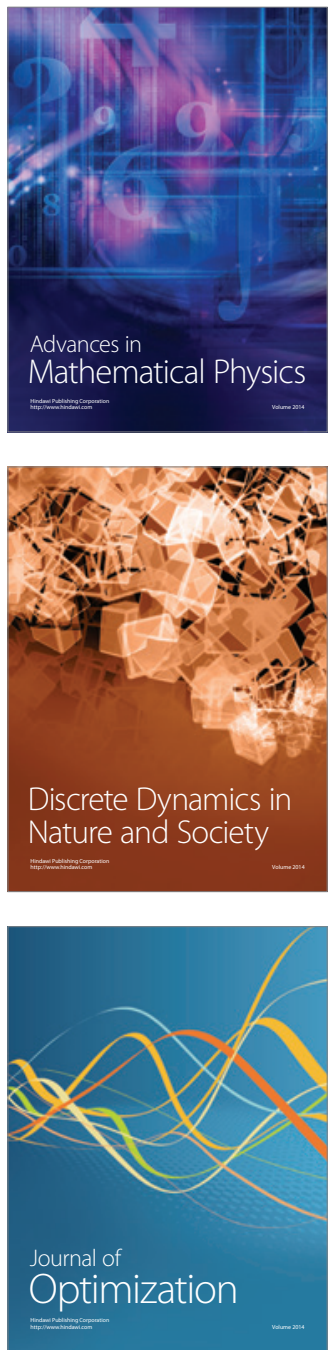